\title{
Automated thermal NDT system applied to internal defects inspection of rolled metal sheets in manufactures
}

\author{
by E. Abramova, $\mathrm{O}$. Budadin and $\mathrm{V}$. Panin
}

Research-and-Production Association of Engineering, Russia, 141350 Moscow region, Khotkovo-1, box 16

\section{Abstract}

This paper is devoted to the description of a new thermal NDT of rolled metal sheet (plate) during manufacturing as well as during movement of the plate with speed up to $2 \mathrm{~m} / \mathrm{s}$, for an iron and steel industry.

Anomalies of the dynamic temperature field arising on the surface of a product are registered in real time by thermovision equipment and are transmitted to a computer.

The defects are revealed by the videoimage analysis of the temperature fields in a computer by means of a special software.

\section{Introduction}

At present, one of the most important problems in the production of flat-rolled goods is arranging for inspection of such products - especially those destined for the use in oil and gas pipelines.

In connection with the recent rise in energy costs and the drop (by as much as $50 \%$ ) in Russian metallurgical production stemming from competition with Western factories, quality standards have become more stringent. An improvement in product quality will also be necessary to satisfy prospective orders for tubes (over 3 million tons of tubing has already been ordered for 1994). Complete inspection of tubular products thus becomes essential, considering that the mechanical strength of pipes used in oil and gas lines, and maximum allowable pressure inside the lines, both depend on the integrity of the constituent metal.

Unfortunately, no such inspection is presently performed at Russian metallurgical plants for the pipes that will be subjected to high pressures in service. Although some factories have foreign-made ultrasonic inspection systems, they cannot be used to inspect all of the tubing in real time (in the mill line).

At Russian steel works plate integrity (available blowholes, spills, cracks, etc.) is often controlled by a selective technique, and the total steel lot quality is judged from the obtained results. Inspection is as a rule performed by a manual shadow method which is low-efficient and labor-consuming.

This problem is the result of several factors: unsatisfactory system productivity; the high temperature of the product under shop conditions (which causes "bubbling" and boiling of the contact medium and thus leads to distortion of the instrument readings); the short distance from the product to the ultrasonic transducer, which makes damaging of the transducer more likely in certain cases (such as looping of the product); the impossibility of incorporating the system into existing rolling technologies without significantly changing the latter.

The Research-and-Production Association of Engineering carried out studies which made it possible to establish the control conditions and to determine the aids which afford inspection of $100-\%$ plate area, real time control, i.e. in accordance with the process, with up to $2 \mathrm{~m} / \mathrm{s}$ plate movement, random plate vibrations, large acoustic noise and plate width variations. Besides, they afford defect detection and marking on steel, and, if necessary, archive data keeping record of them. 
The analysis of existing nondestructive testing methods showed that thermal control is optimal in terms of the best accomplishment of requirements or control conditions. The essence of the method is as follows. Dynamic (varying with time) temperature field is formed on the surface of the web, for example, plate with local defects.

\section{Theoretical aspects}

The temperature field of the surface of the product is a source of information on heattransfer characteristics, these characteristics in turn being affected by the presence of defects - pipe, cracks, laminations, unfused regions, foreign inclusions, etc. Such defects influence the temperature field of the product surface by creating local temperature gradients, which are due to the difference in the thermophysical properties of the sound and defective sections of the product.

Here field characteristics, in particular, anomalies, will depend on defects and defect parameters (length $S$, depth $h$, and opening $\delta$ ). When analysing the plate temperature field one can draw a unique conclusion on defect detectability based on the analysis of the temperature field by means of special procedures elaborated in the Research-and-Production Association of Engineering.

The theoretical studies of quantitative parameters of thermal control were performed on the model [1]. The investigated object was a $12 \mathrm{~mm}$-thick steel plate; the results are given in Fig.1. The control results are better if the plate gage is less than $12 \mathrm{~mm}$.

When analyzing the plots, the following conclusions can be drawn. The bottom side temperature change $\Delta t_{b}$ does not depend virtually on the defect depth $h$ : when $h$ is changed by a factor of 10 (from 1 to $10 \mathrm{~mm}$ ), $\Delta t_{b}$ is changed only by a factor of 3 (from $13.5^{\circ}$ to $16^{\circ} \mathrm{C}$ ). Meanwhile a greater dependence of $\Delta 4$ on $h$ is noted for the top side. An effect of virtually linear saturation of $\Delta t$ from $S$, with $\Delta t$ above $8^{\circ} \mathrm{C}$, is observed at $S \geq 5 \mathrm{~mm}$. An effect of sharp saturation of $\Delta t$ from $\delta$ occurs at $\delta>0.05 \mathrm{~mm}$, i.e., during plate inspection the defects behave like ideal thermal insulators. The dependence of $\Delta t_{t}$ and $\Delta t_{b}$ on $Q$ and $\tau_{b}$ is linear. The optimal lag time is in the range of $\tau_{\lambda}=1.5-3.5 \mathrm{~s}$.

Figure 2 shows the generalized dependence which allows heating parameters to be defined if the required $\Delta t$ must be provided.

The obtained results show that the thermal method affords a true detection of $5 \times 5 \mathrm{~mm}$ and more air defects with the opening $\delta>0.05 \mathrm{~mm}$ at $Q=350 \mathrm{~kW} / \mathrm{m}^{2}, \tau_{\mathrm{b}}=0.5 \mathrm{~s}, \tau_{\lambda}=1.5-3.5 \mathrm{~s}$.

The confidence criterion of defect detection was taken to be $\Delta t=8^{\circ} \mathrm{C}$ which, with a temperature resolution of up-to-date thermal analysers of $\Delta t=0.5^{\circ} \mathrm{C}$, involves a signal-to-noise ratio of 16. It is quite sufficient in practice. The empirical formula can be introduced for easier calculation

$$
\Delta t_{b}=\Delta t_{0} \frac{\mathrm{Q}_{\tau_{\mathrm{b}}} S}{\mathrm{Q}_{\mathrm{O}_{\tau_{\mathrm{b} 0}}} S_{0}}
$$

where parameters with the index 0 correspond to minimal detected defect.

The theoretical calculations were proved by experimental studies which were conducted on a nondestructive testing rig [2]. The studied objects were specimens prepared from $400 \times 400 \times 12 \mathrm{~mm}$ sheets of steel St 3 . Holes were made in some of the specimens to simulate defects (holes of different diameters extending to different (to $12 \mathrm{~mm}$ ) depths, with washers at the bottom of each hole and press-fitted end-pieces). The specimens also had natural defects in the form of central and edge cracks. Investigators also studied bimetallic rolled and welded

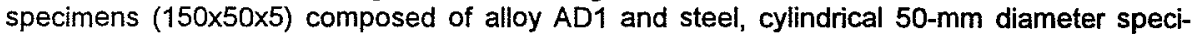


mens of alloy D16T with lateral holes $0.4-1.4 \mathrm{~mm}$ in diameter and $2 \mathrm{~mm}$ in depth, and $20 \mathrm{~mm}$ diameter Ti-Si specimens joined with a soft solder.

X-ray (on a RUP-150 unit) and ultrasonic (on a Krautkrămer unit) inspections were performed simultaneously with the thermal inspections. The quality of the adhesive joint in the cold-rolled bimetallic products was first checked by the thermal method. It was then further checked by physically separating the cladding layer from the base layer and comparing the fractographs with the thermographs. The thermal inspection was performed on a laboratory unit consisting of a "Raduga-5" thermal analyzer, a computer, a heater, and special interfaces with software. The experimental results confirmed the theoretical conclusions. Fig. 3 shows the heat pattem of a defective specimen.

\section{Experimental configuration}

Based on the theoretical and experimental results and judging from inspection requirements, technical aids for the thermal plate control were developed. The block diagram is shown in Fig.4. The diagram specification is as follows (the whole plate area is inspected in a remote way): frame input time $0.05 \mathrm{~s}$; data processing time with output of the result $0.95 \mathrm{~s}$; minimal detected defect $5 \times 5 \mathrm{~mm}$; heating source power $350 \mathrm{~kW} / \mathrm{m}^{2}$.

A trigger signal is given to the computer from the sensor at the start of the sheet movement. On this signal the computer initiates the heat generator control unit which activates the thermal generator with simultaneous invocation of temperature field input and processing software. Within an optimal lag time, the heated plate section is transferred to the temperature field re-

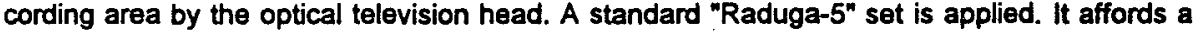
$17.5^{\circ} \times 20^{\circ}$ field of view which allows the whole surface to be controlled, at $2 \mathrm{~m}$-distance of the optical head from the plate surface. Using application software, through a special controller the computer provides a virtually instantaneous (within $0.05 \mathrm{~s}$ ) recording of the temperature field of the viewed area with a 1-s scan. The time $(0.95 \mathrm{~s})$ between the recording end of the previous frame and the beginning of the next frame is used for information processing: image processing preparation, defect detection, automatic control of technical aids, archiving and support. On defect detection, the computer initiates the control unit of the defect marking system and records the plate defects through it.

The analyzer system recorded the temperature field of the specimen surface at a rate of 25 frames a second (4000 lines, 135 elements per line). The linear dimensions of a frame in the tests conducted with ferrous metals is $2 \times 2 \mathrm{~m}$. The linear displacement of the specimen during the line-by-line scanning was $0.5 \mathrm{~mm}$ (for a specimen velocity of $2 \mathrm{~m} / \mathrm{sec}$ ). This was appreciably greater than the specified sensitivity $(10-20 \mathrm{~mm})$. Two-hundred lines are sufficient to obtain information from a $2 \times 2 \mathrm{~m}$ object with a spacing of $10 \mathrm{~mm}$. A total of $0.05 \mathrm{sec}$ is needed to read the $2 \times 2 \mathrm{~m}$ frame, the remaining 0.95 sec being sufficient for the computer to analyze the information and make a decision. The number of elements in a line (135) provides for a sensitivity of $13 \mathrm{~mm}$ with respect to the $y$-axis, which also satisfies the specifications.

The specimens were heated to $40-70^{\circ} \mathrm{C}$ in a thermostat and subjected to sudden additional heating by the heater over a period of $0.1 \mathrm{sec}$. Investigators then recorded the temperature field of the surface (in the case of impulsive heating, after 2-4 sec) and analyzed the information on the computer.

The studies confirmed the presence of all of the defects initially detected by clients using $x$ ray and ultrasonic methods. Other defects that were not found by these methods were also discovered.

The time specified for inspection of a $2 \times 2 \mathrm{~m}$ product made of a ferrous metal is no more than 1 sec. It took 30 sec to inspect specimens of alloy D16T and print out the results, which is much shorter than the time required by the ultrasonic method (10 min). 
The sensitivity of the thermal method is $0.4 \mathrm{~mm}$ at a depth of $2.5 \mathrm{~mm}(5 \mathrm{~mm}$ at a depth of $120 \mathrm{~mm}$ ), while the $x$-ray and ultrasonic methods are able to detect holes 1.4 and $0.8 \mathrm{~mm}$ in diameter, respectively.

Thus, the superior sensitivity of the thermal method permits its use on rolled products moving at a speed of up to $2 \mathrm{~m} / \mathrm{sec}$. In contrast to the ultrasonic and $x$-ray methods, the thermal method requires no coupling medium (is "dry"). This simplifies the inspection process considerably. In addition, mechanical scanning systems are replaced by more reliable electromechanical systems.

Of particular importance in metallurgy is checking for near-surface defects in regions which turn out to be "dead zones" when ultrasound is used (edge effect). Moreover, the quality of the metal in these sections (which are roughly $3-5 \mathrm{~mm}$ wide) in large part determines the quality of welds on tubes. The thermal method makes it possible to find also discontinuities in these zones.

Now the Research-and-Production Association of Engineering, jointly with the North steel Joint-Stock Co. and the Metallurgprom Concern, completed the basic theoretical and experimental studies which proved a feasible development of the thermal control system of plate integrity for the cutting area of No. 2 plate/strip shop, which conforms with the technical requirements of the North steel Joint-Stock Co. Comparative studies showed the advantages of the thermal method over the ultrasonic technique in terms of production rate, response, reliability, and performance.

The use of the above-described method will not only make it possible for domestic plants to compete successfully with foreign companies on the world market, but will also avoid losses from accidents in oil and gas pipelines due to poor-quality metal and defective welds.

\section{REFERENCES}

[1] RAPOPORT (D.A.) and BUDADIN (O.N.). - Model of automated nondestructive active thermal control.- Defectoskopia, 1983, No.12.

[2] PADALKO (G.A.), SLIVA (S.S.), FOMENKO (V.K.) et al. - "Raduga-5". Thermal vision plant for nondestructive testing.- Defectoskopia, 1990, No.7, p.76-83. 


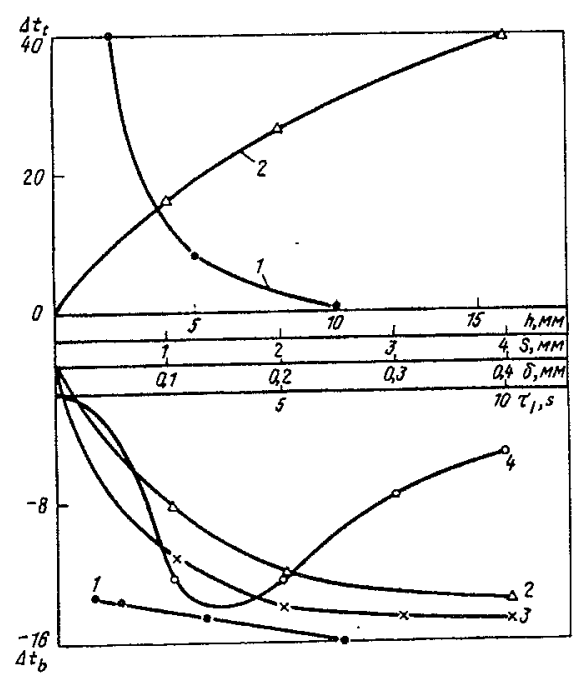

Fig.1. Dependences of temperature differences between defectless and defective portions of plate on the heating side $\left(\Delta t_{t}\right)$ and the opposite side $(\Delta t(M V>b<D>): 1$ - defect depth (h), 2 - defect length (S), 3 - opening ( 8 ), 4 - temperature lag record time $\tau_{1}$

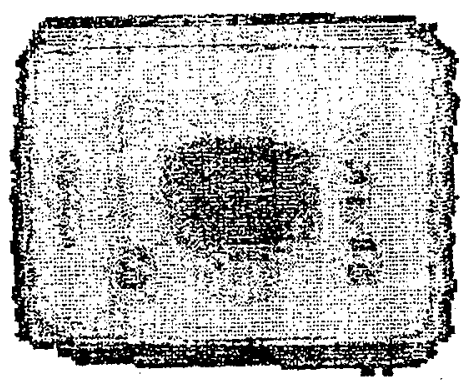

Fig.3. Heat pattern of defective specimen; $t_{1}=2.5 \mathrm{~s}$, $\mathrm{Q}=100 \mathrm{~kW} / \mathrm{m}^{2}, \tau_{\mathrm{b}}=1 \mathrm{~s}, \Delta \mathrm{t}_{\mathrm{b}}=4.5^{\circ} \mathrm{C}$ by calibrated scale

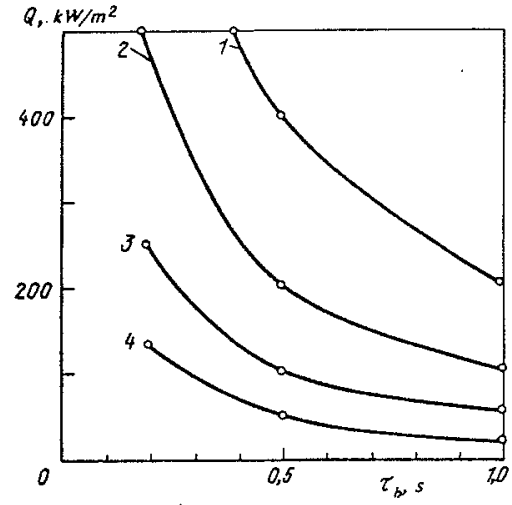

Fig.2. Dependence of heating source power $(Q)$ on heating time $\left(\tau_{b}\right): 1-\Delta t_{b} 10^{\circ} \mathrm{C}, 2->5^{\circ} \mathrm{C}, 3->2.5^{\circ} \mathrm{C}, 4->1.25^{\circ} \mathrm{C}$

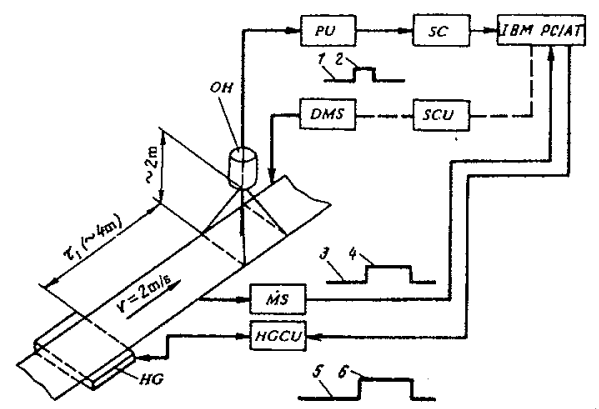

Fig.4. Block diagram of thermal plate vision facilities: $\mathrm{OH}$ optical head of thermal vision set, PU - electric signal processing unit, SC - special controller, DMS - defect marking system, SCU - control unit of defect marking system, MS - movement sensor, HGCU - heat generator control unit, HG - heat generator, 1, 2 - defect: no, yes, 3, 4 - movement: no or yes, 5, 6 - heating: no or yes 